\title{
Highlights from the 2018 European Respiratory Society International Congress: sleep and clinical physiology
}

\author{
Isaac Almendros (1) 1,2,14, Sébastien Baillieul (103,4,5,14, Candela Caballero 2,6,14, \\ Luiza Helena Degani-Costa (1078,14, Michael Furian 9, 14, Camila Hirotsu (10)14' \\ Zafeiris Louvaris ${ }^{11,14}$, Sira Thiel ${ }^{9,14}$ and Christopher D. Turnbull ${ }^{12,13,14}$
}

Affiliations: 'Unitat de Biofísica i Bioenginyeria, Facultat de Medicina i Ciències de la Salut, Universitat de Barcelona, Barcelona, Spain. ${ }^{2}$ Centro de Investigación Biomédica en Red de Enfermedades Respiratorias, Madrid, Spain. ${ }^{3} \mathrm{HP} 2$ laboratory, Grenoble Alpes University, Grenoble, France. ${ }^{4}$ U1042, INSERM, Grenoble, France. ${ }^{5}$ Pôle Thorax et Vaisseaux, Grenoble Alpes University Hospital, Grenoble, France. ${ }^{6}$ Instituto de Biomedicina de Sevilla, Hospital Universitario Virgen del Rocío/CSIC/Universidad de Sevilla, Seville, Spain. ${ }^{7}$ Pulmonary Function and Clinical Exercise Physiology Unit, Pulmonary Division, Dept of Medicine, Federal University of São Paulo, São Paulo, Brazil. ${ }^{8}$ Hospital Israelita Albert Einstein, São Paulo, Brazil. ${ }^{9}$ Dept of Pulmonology and Sleep Disorders Center, University Hospital of Zurich, Zurich, Switzerland. ${ }^{10}$ Center for Investigation and Research in Sleep, University Hospital of Lausanne, Lausanne, Switzerland. ${ }^{11}$ Faculty of Movement and Rehabilitation Sciences, Division of Respiratory Rehabilitation, Dept of Rehabilitation Sciences, KU Leuven, Research Group for Rehabilitation in Internal Disorders, University Hospitals Leuven, Leuven, Belgium. ${ }^{12}$ NIHR Oxford Biomedical Research Centre, University of Oxford, Oxford, UK. ${ }^{13}$ Oxford Centre for Respiratory Medicine, Oxford University Hospitals NHS Foundation Trust, Oxford, UK. ${ }^{14}$ All authors contributed equally and are listed alphabetically.

Correspondence: Isaac Almendros, Unitat de Biofísica i Bioenginyeria, Facultat de Medicina i Ciències de la Salut, Universitat de Barcelona, Casanova 143, 08036 Barcelona, Spain. E-mail: isaac.almendrosđub.edu

ABSTRACT The 2018 European Respiratory Society (ERS) International Congress held in Paris, France, served as a platform to discover the latest research on respiratory diseases, the improvement in their treatments and patient care. Specifically, the scientific sessions organised by ERS Assembly 4 provided novel insights into sleep disordered breathing and fresh knowledge in respiratory physiology, stressing its importance to understanding and treating respiratory diseases. This article, divided by session, will summarise the most relevant studies presented at the ERS International Congress. Each session has been written by early career members specialised in the different fields of this interdisciplinary assembly.

@ERSpublications

A selection of the best sessions from Assembly 4 at the \#ERSCongress 2018 by @EarlyCareerERS http://bit.ly/2Wot0CJ

Cite this article as: Almendros I, Baillieul S, Caballero C, et al. Highlights from the 2018 European Respiratory Society International Congress: sleep and clinical physiology. ERJ Open Res 2019; 5: 00201-2018 [https://doi.org/10.1183/23120541.00201-2018]. 


\section{Pathophysiological aspects of sleep disordered breathing: intermittent hypoxia}

Obstructive sleep apnoea (OSA) has been associated with a wide variety of cardiovascular, neurocognitive and metabolic consequences. Intermittent hypoxia (IH), a hallmark of OSA, has been suggested to play an important role in the development of cardiovascular disease. Proposed mechanisms include sympathetic activation, oxidative stress and systemic inflammation.

Moulin et al. [1] explored the role of hypoxia inducible factor-1 (HIF-1) signalling in IH-induced cardiovascular injury. They took HIF- $1^{+/-}$and wild-type mice, and exposed them to 21 days of either $\mathrm{IH}$ or normoxia. The marked effects of $\mathrm{IH}$ on mitochondrial respiration, reactive oxygen species production and calcium retention capacity were all blocked in the HIF- $1^{+/-}$mice, thus suggesting a central role of HIF-1 signalling in IH-mediated cardiovascular disease.

DÉTRAIT et al. [2] explored the role of 12 weeks of IH in the development of ischaemic cardiomyopathy following left coronary artery ligation in rats. They showed that IH leads to cardiac hypertrophy, cardiac fibrosis and impaired cardiac function. In parallel, they showed marked sympathetic activation with $\mathrm{IH}$ and impaired calcium homeostasis, suggesting their central roles in IH-mediated cardiovascular disease.

TuRnbull et al. [3] explored the effects of IH on leukocyte gene expression from OSA patients undergoing continuous positive airway pressure (CPAP) withdrawal with and without supplemental oxygen. Patients previously treated with CPAP were withdrawn onto 14 nights of supplemental oxygen $\left(5 \mathrm{~L} \cdot \mathrm{min}^{-1}\right)$ or sham in a crossover randomised controlled trial (RCT). Turnbull et al. [3] identified several genes that were only upregulated with CPAP withdrawal onto sham, suggesting that gene changes in circulating leukocytes were IH dependent. The authors also showed activation of key inflammatory pathways, suggesting a role for NF-אB-mediated inflammation in IH-mediated cardiovascular disease in OSA.

Following with the mechanisms linking IH to cardiovascular disease, THIEL et al. [4] explored the impaired cerebral vascular reactivity in response to 14 nights of CPAP withdrawal compared to continuation of real CPAP in moderate to severe OSA patients. Although there was a significant increase in blood pressure upon CPAP withdrawal and a full recurrence of sleep disordered breathing, there was no significant effect of CPAP withdrawal on cerebral vascular reactivity (CVR) assessed via blood oxygen level-dependent magnetic resonance imaging (MRI). Daytime CVR did not show any significant reduction after 2 weeks of CPAP withdrawal, implying that CVR regulation outlasts other pathophysiological effects of OSA in the short term.

SuAREZ-Giron et al. [5] demonstrated that aspirin, a cyclo-oxygenase inhibitor, was able to inhibit IH-induced aortic damage, including intima-media thickness, elastin disorganisation, elastin fragmentation, length between fragmented fibre endpoints, aortic wall collagen abundance and mucoid deposition. These results suggest that aspirin should be prospectively evaluated as adjuvant treatment in patients with OSA. In a second work, Almendros et al. [6] evaluated the specific role of IH and sleep fragmentation in the progression of Alzheimer's disease. The authors found that sleep fragmentation increases amyloid- $\beta$ deposition in the hippocampus of a mouse model of Alzheimer's disease, when compared to sleep control animals. However, IH had no significant effect on amyloid plaque deposition, either in the cortex or in the hippocampus.

TrZePizur et al. [7] explored the potential link between OSA and liver injury. Liver stiffness was assessed using the FibroScan (Echosens, Paris, France), as this is a robust and noninvasive physical marker of liver fibrosis. They showed that severe OSA (apnoea-hypopnoea index (AHI) $>30$ events per $\mathrm{h}$ ) was present in $71.9 \%$ of patients with a high liver stiffness measurement (LSM). After adjustment for age, sex, metabolic syndrome and HOMA-IR index (homeostatic model assessment, insulin resistance), severe OSA remained highly and independently associated with significantly high LSM, leading to the assumption that this might predispose OSA patients to a higher risk of liver fibrosis.

REvol et al. [8] analysed, in VigiBase, the reported cases of sleep apnoea syndrome (SAS) among ticagrelor users compared to other antiplatelet agents. The reporting odds ratios for ticagrelor were significant for SAS and for dyspnoea but not for the other antiplatelet agents employed in the study (clopidogrel, prasugrel and aspirin). Revol et al. [8] suggested that ticagrelor can induce respiratory drive deregulations that might explain the occurrence of dyspnoea and SAS.

\section{Epidemiology, phenotypes and cardiometabolic comorbidities of OSA}

In Europe, the prevalence of OSA is still unclear. MALHOTRA et al. [9] developed an algorithm of AHI conversion to estimate the prevalence of OSA for each European country using the most recent scoring criteria. From 39 European countries, a prevalence of $44.0 \%$ was found for AHI $\geqslant 5$ events per $h$ and of $22.7 \%$ for AHI $\geqslant 15$ events per $\mathrm{h}$. Globally, this leads to a prevalence of 936 million and 424 million people, respectively. These impressive numbers raise a need to increase awareness of OSA, and 
to develop new diagnostic and therapeutic approaches that may consider the different phenotypes of OSA.

In the era of precision medicine, it is important to discuss the limitations of AHI in the assessment of the diverse spectrum of OSA. To characterise the heterogeneity of OSA into well-defined phenotypes may improve OSA prognosis and lead to personalised treatments [10]. However, this is a challenging task since there is still no consensus of how it should be accomplished. Of note, recent cross-sectional studies have shown the existence of three symptom-based cluster designations of OSA (disturbed sleep, minimally symptomatic and excessively sleepy) in different samples [11-14]. Using data from the European Sleep Apnoea Database (ESADA) ( $\mathrm{n}=17325$ adult patients, 29.9\% women), ANTTALAINEN et al. [15] assessed four OSA phenotypes based on the presence and absence of excessive daytime sleepiness (EDS) and/or insomnia symptoms. The phenotype with insomnia alone was the most common in the total sample, especially in women. However, the association with comorbidities differed among phenotypes. OSA patients with insomnia alone had a higher prevalence of cardiovascular disease, and those with both insomnia and EDS showed higher prevalence of metabolic, pulmonary and psychiatric outcomes. Increased nadir oxygen saturation was an independent protective factor only in the OSA phenotypes that presented insomnia, corroborating previous literature [16], which suggests that the improvement of insomnia symptoms may reduce nocturnal hypoxaemia.

In coronary artery disease (CAD) patients, OSA is much more prevalent but the role of its phenotypes is still unknown, although relevant for disease management. Thus, PEKER et al. [17] presented a secondary analysis of the RICCADSA trial (Randomized Intervention with CPAP in Coronary Artery Disease and Sleep Apnoea), comparing the sleepy and nonsleepy OSA phenotypes. The results showed that the presence of sleepiness (Epworth Sleepiness Scale (ESS) score $\geqslant 10$ ) in OSA patients with CAD was associated with increased total sleep time and oxygen desaturation index (besides the same level of AHI) but lower levels of NT-proBNP, which was negatively correlated with slow-wave sleep, ESS score and total sleep time. In another subset analysis of this cohort, the BALCAN et al. [18] focused on the effects of CPAP treatment on depressive symptoms using the same rationale of sleepy and nonsleepy OSA phenotypes. At baseline, depressive symptoms were associated with higher ESS scores. However, CPAP treatment was effective in improving depressive mood in both sleepy and nonsleepy OSA phenotypes after 3 months. The improvement remained significant at the 12-month follow-up, being predicted by CPAP adherence, sex, body mass index (BMI) and reduction of subjective sleepiness.

Although there is a consensus that the treatment of OSA leads to a small decrease in blood pressure [19], prospective epidemiological evidence is scarce and divergent, with some positive $[20,21]$ and negative $[22,23]$ results from community samples. Thus, Hirotsu et al. [24] assessed the sleep determinants of incident hypertension in an unselected general population sample of the HypnoLaus cohort, which was followed up over 5 years. In 1141 individuals free of hypertension at baseline, the authors found that less consolidated sleep, with lower $\delta$ and $\sigma$ power in non-REM sleep, as well as a worse vascular reactivity characterised by longer pulse wave amplitude (PWA) drops and less PWA frequency during sleep, were independently associated with a higher risk of developing hypertension. Although in unadjusted analyses, mild and moderate to severe OSA as well as hypoxia-related sleep features were associated with incident hypertension, these results were no longer significant after adjustment for BMI.

However, results from the ESADA cohort showed that mild OSA patients $(n=2457)$ had increased odds ratio for the prevalence of hypertension compared to non-OSA snorers $(n=2517)$ [25]. Besides the difference in the study design (cross-sectional versus prospective) and population (clinical versus general), the definition of hypertension also differed between the two studies. In ESADA, it was based on self-report of hypertension and/or use of antihypertensive medication, while in HypnoLaus cohort, the standard average of the two last measurements of systolic and diastolic pressure $\geqslant 140 / 90 \mathrm{mmHg}$ combined with the use of antihypertensive medication was used.

Finally, the last presentation of this session answered the unsolved question of the relationship between OSA and metabolic syndrome (MetS). Using two prospective general population samples (Episono from Brazil and HypnoLaus from Switzerland), Hirotsu et al. [26] showed for the first time, in a merged population of 1853 individuals free of MetS at baseline, that moderate to severe OSA was independently associated with a doubled risk of developing MetS. Using mediation regression analysis, the authors demonstrated that OSA increased the number of MetS risk factors through mediation of nocturnal hypoxia, leading to increased waist circumference and glucose levels. However, when assessing the reverse relationship in a subset analysis of Episono cohort, the presence of MetS was not associated with the development of new cases of OSA after control for BMI. This new evidence is a proof of concept of the causal relationship between OSA and MetS, and thus RCTs will be necessary to conclude whether the treatment of OSA will reduce the incidence of MetS. 
State of the art session: sleep and breathing disorders

Clinical dyspnoea: brain mechanisms and their clinical impact on brain functions

Treating breathlessness via the brain

Dyspnoea is the main symptom in prevalent chronic diseases. This symptom debilitates millions of people with chronic illness; it is all consuming and life changing. The perception of dyspnoea has a strong influence on health behaviour and the course of disease. The presence of dyspnoea impacts the brain not just as an emotional and behavioural factor, but also acting on cognition, changing the way brain works. Previous studies have shown that dyspnoea is associated with various types of brain activation and deactivation. In healthy individuals, there is no activation of the premotor cortex during quiet breathing $[27,28]$. However, in amyotrophic lateral sclerosis patients with severe respiratory muscle weakness, an activation premotor cortex respiratory-related cortical activity was observed [29], while during noninvasive ventilation (NIV), this activity was attenuated [30], suggesting that this activation appears in the presence of a mechanical inspiratory load. The activation of the brain does not appear only when dyspnoea is presented, but also when anticipating the problem. The anticipation of this aversive symptom induces fear in many patients, resulting in negative and unhealthy behaviours leading to an increasingly sedentary lifestyle. A study carried out in healthy individuals that provoked dyspnoea by using resistive-load breathing during functional MRI showed that dyspnoea anticipation activated some brain areas involved in dyspnoea perception [31].

Additionally, a recent study showed that observing dyspnoea in others causes dyspnoea and increases brain responses in the absence of respiratory changes, suggesting the negative effect that dyspnoea might have on the family and caregivers of dyspnoeic patients [32]. Notably, the perception of breathlessness is not related to objective lung function in chronic respiratory diseases such as chronic obstructive pulmonary disease (COPD) [33].

Various approaches to relieving dyspnoea without affecting lung function have been described. In a study that analysed the effect of rehabilitation in dyspnoea perception and the changes produced in the brain in COPD patients, it was shown that pulmonary rehabilitation was associated with altered neural responses, which could influence breathlessness perception [34]. Rehabilitation in these patients improved both dyspnoea and psychological symptoms such as anxiety. Psychological symptoms, such as anxiety and depression, are very common in COPD and are related to various negative health outcomes, including dyspnoea. COPD patients with high anxiety levels report more dyspnoea at rest, during exercise and during pulmonary rehabilitation. Acting on anxiety level using mindfulness therapy has been proposed to improve dyspnoea perception in COPD patients [35].

Hypoxia, arousals and breathing effort: a roadmap to comorbidities?

OSA and cognition

As previously stated, the relationship between OSA and cardiovascular and metabolic disease has been well established [5, 36-38]. However, in recent years, evidence linking OSA with other comorbidities, such as degenerative neurological disease and associated cognitive impairment, has emerged [39]. During the ERS Congress in Paris, Drs Rosenzweig and Ryan reviewed this emerging field, and explained the mechanism implicated in this associations. The cognitive domains that can be affected in OSA patients are psychomotor speed, attention, memory, executive function (higher order cognitive skills such as planning, problem solving and mental flexibility) and social cognition. $\sim 80 \%$ of OSA patients complain about excessive daytime sleepiness and cognitive impairments, and $50 \%$ of patients report personality changes [40]. Additionally, an association has been described between major depressive disorder, anxiety and post-traumatic stress disorder. In addition, difficulty in interpersonal relationships, irritability, decreased work and school efficiency, and car accidents have all been documented in OSA patients. More recently, the results of a meta-analysis suggest that patients with Alzheimer's disease may have five times higher risk of presenting OSA, compared to individuals of similar age without cognitive impairment [41]. Furthermore, it has been reported that individuals with OSA may develop mild cognitive impairment and Alzheimer's disease at a younger age [42], and OSA treatment with CPAP delays the age of onset of mild cognitive impairment.

\section{Exercise is medicine: testing and treating patients with some effort}

Exercise intolerance is common in COPD [43]; however, the underlying pathophysiology is multifactorial and varies between patients [44]. In COPD Global Initiative for Chronic Obstructive Lung Disease (GOLD) stage 2-4, improvement in endurance with NIV, compared to pursed-lip breathing, during an interval cycle exercise was achieved. This was associated with reduced dynamic hyperinflation, perceived dyspnoea and leg discomfort [45]. Improved exercise capacity in COPD patients might have also a beneficial effect on the increased risk of falling in COPD [46]. ReYNAUD et al. [47] proposed a novel cut-off value of the Timed Up and Go (TUG) test of $11 \mathrm{~s}$ to detect fallers in otherwise stable COPD patients. 
In comparison to this value, $13.5 \mathrm{~s}$ is proposed in elderly patients $>65$ years of age [48] and according to a recent meta-analysis, in contrast to the good sensitivity and specificity in COPD patients, the predictive value is poor in the healthy elderly [49]. In COPD patients, predictors for worse TUG performance were COPD severity, low quadriceps strength or a decreased 6-min walk distance (6MWD).

Most people living with asthma can experience exercise-induced bronchoconstriction (EIB). However, EIB is not exclusively present in people with asthma and a prevalence of $20 \%$ has been estimated in the absence of asthma [50]. According to the American Thoracic Society guidelines released in 2013 and the later update in 2016, symptoms suggestive of EIB require further evaluation through exercise challenge or other indirect bronchial provocation tests $[51,52] . A \geqslant 10 \%$ fall in forced expiratory volume in $1 \mathrm{~s}\left(\mathrm{FEV}_{1}\right)$ after an exercise challenge is used for diagnosis. Pharmacological treatments of EIB include symptom prevention, such as inhalation of short-acting $\beta_{2}$-agonists or anticholinergic agents. The presence of different endotypes has been suggested to be responsible for the high variability in individual responses to bronchodilators prescribed to prevent EIB. Two distinct inflammatory endotypes have been describe: the T2-high endotype, characterised by eosinophilic inflammation; and the T2-low endotype, characterised by neutrophilic inflammation. Bonini et al. [53] found that T2-endotype influences bronchodilator response in EIB. Specifically, they found no protective effect on EIB in the T2-high group from inhalation of the anticholinergic ipratropium compared to inhalation of the $\beta_{2}$-agonist salbutamol. To investigate whether the forced oscillation technique (FOT) [54] could produce equivalent measures of airway calibre post-exercise challenge to maximal expiratory manoeuvres, SECCOMBE et al. [55] compared both techniques to identify patients with EIB independent of bronchial hyperresponsiveness (BHR) in asthma patients with and without BHR versus controls. The FOT parameters resistance and reactance at $5 \mathrm{~Hz}$ oscillation frequency correlated well with post-exercise FEV1 manoeuvres, and might serve as a tool to distinguish asthmatic patients with EIB from those with BHR and as an alternative to post-exercise FEV1 manoeuvres.

In contrast to EIB, breathing complaints during exercise that relieve within a few minutes after exercise termination are characteristic for exercise-induced inspiratory symptoms, i.e. exercise-induced laryngeal obstruction (EILO). Continuous laryngoscopy exercise (CLE) testing is the gold standard for diagnosing EILO. However, this intervention could influence the maximum gas exchange values achieved. HAMMER et al. [56] reported no differences in exercise performance between maximal treadmill cardiopulmonary exercise testing (CPET) with and without CLE in regard to maximal oxygen uptake in healthy athletes. A novel approach to EILO was introduced during the ERS Congress: a proof of concept study using laser surgery reducing supraglottic obstruction. This resulted in better post-surgery exercise performance and lower perceived dyspnoea in two subjects with severe EILO compared to pre-surgery symptom-limited CPET [57].

Another study in patients with excessive dynamic airway collapse showed improved 6-min walk performance when applying CPAP compared to subtherapeutic CPAP therapy [58]. In contrast to short-term improvements, EDVARSEN et al. [59] investigated whether 20 weeks of high-intensity training after surgery has an effect on 5-year survival in patients with lung cancer. Although no differences were reported in the 5-year survival, they found that the training group lived, on average, several months longer than the control group.

\section{Respiratory muscles at rest, during exercise and in the critically ill patient}

Beyond lung function, respiratory muscle dysfunction (RMD) is currently considered a major comorbidity in COPD. In fact, RMD can modulate prognosis, exercise tolerance and quality of life in COPD patients [60]. In COPD, even though respiratory muscles may show some positive adaptations induced by the increased respiratory overload $[60,61]$, they also have reduced endurance $[62,63]$ and strength, presenting only $59 \%$ of inspiratory and 69\% expiratory muscle strength compared with control values [64]. This goes beyond the level of impairment observed in skeletal muscle in those patients ( $75 \%$ of control values for lower limb and 78\% for upper limbs) [64]. Frequent exacerbations [65] negatively impact muscle mass and function in these patients. Thus, the cross-sectional area of intercostal muscles was significantly reduced by $14 \%$ in fragile COPD patients compared to controls [66]. Moreover, hypoxia and oxidative stress seem to play a determinant role [67] in the phenotypic and functional muscle modifications [63], which are probably underpinned by epigenetic modifications $[68,69]$.

The exercise limitation caused by RMD [70] and the increased work of breathing as a result of dynamic hyperinflation [71] contribute to the uncomfortable feeling of breathlessness [72]. Rather than a pure structural muscle impairment, RMD must be considered as a neuromuscular dysfunction [72]. In terms of the diaphragm, in COPD patients, the increased work of breathing and the reduced muscle capacity can lead to an increased neural respiratory drive (NRD) even at rest [73]. Thus, NRD at rest was significantly increased in COPD patients compared to healthy subjects [74]. During exercise, NRD in moderate to 
severe COPD patients is characterised by neuroventilatory uncoupling [75] and is associated with breathlessness intensity [76]. Interestingly, diaphragm electromyogram expressed as a percentage of maximum is also a strong predictor of dyspnoea in fibrotic interstitial lung disease [77]. Thus, it has been proposed as a useful physiological biomarker for breathlessness in COPD [76] and in fibrotic interstitial lung disease [77].

It has been reported that $64 \%$ of patients within $24 \mathrm{~h}$ following their admission to the intensive care unit (ICU) can develop diaphragm dysfunction [78]. In addition, a more recent prospective longitudinal study has reported that this percentage can be increased up to $79 \%$ in critically ill patients receiving prolonged mechanical ventilation at some point during their ICU stay [79]. Diaphragm dysfunction can be developed as a consequence of multiorgan failure, critical illness polyneuropathy and/or myopathy, hypercatabolism or prolonged mechanical ventilation [80]. The association of diaphragm dysfunction with a poorer prognosis [78], a higher rate of mechanical ventilation weaning failure and an increased duration of mechanical ventilator [79], has increased its interest in intensive care.

Diaphragm weakness can be assessed by measuring the thickening fraction of the diaphragm by ultrasonography [81] and has recently shown similarly strong performance to twitch tracheal pressure in response to magnetic phrenic stimulation in the prediction of failure of the spontaneous breathing trial [82]. In severe acute exacerbation of COPD, ultrasonography assessment of diaphragm dysfunction is a reliable and accurate tool to identify patients at major risk of NIV failure and worse prognosis [83].

Inspiratory muscle training (IMT) is of great interest to prevent RMD. In COPD, it has been suggested that IMT must be combined with general exercise training (GET), which increases ventilatory pump capacity and provides a reduction in ventilatory requirements. Two recent RCTs assessed the effects of 3 [84] or 4 weeks [85] of IMT combined with GET, in comparison to sham IMT combined with GET [84], or GET alone [85]. Both failed to show any improvement in 6MWD or health-related quality of life outcomes, despite improvements in maximal inspiratory pressure [84]. A 12-week RCT conducted in COPD patients with inspiratory muscle weakness assessed the effects of IMT versus sham IMT both combined with GET on inspiratory muscle function, exercise capacity and health-related quality of life [86]. In contrast to $6 \mathrm{MWD}$, some improvements were observed in patients with marked inspiratory muscle weakness on the endurance cycling test. In acute conditions, particularly in the ICU, IMT has been shown to be an adjunct to the treatment of patients with mechanical ventilation weaning difficulty [87]. Further research is still ongoing, including a RCT (www.clinicaltrials.gov identifier number NCT03240263) to evaluate the effects of a novel IMT method on weaning outcomes in selected patients with weaning difficulties [88].

\section{Mechanisms that underlie exercise limitation in various respiratory diseases}

The increase in sympathetic vasoconstrictor outflow to the limb muscles upon activation of a metaboreflex triggered by respiratory muscle fatigue has been proposed as one potential mechanism limiting whole-body exercise capacity in COPD patients [89]. JoHnson et al. [90] investigated whether this mechanism may limit single-leg knee extensor exercise tolerance. They showed that following maximal volitional hyperpnea, single-leg knee extensor exercise tolerance was lower compared to exercise conditions without imposing respiratory muscle fatigue. Nevertheless, the decrease in exercise tolerance was not associated with greater leg muscle fatigue after exercise. Authors concluded that the reduction in single-leg exercise tolerance because of prior fatigue of the respiratory muscle was not via acceleration of peripheral muscle fatigue secondary to activation of respiratory muscle metaboreflex, but due to perceptual responses, as patients demonstrated greater perception of effort. Evidently, these results open new perspectives and challenges.

Regarding pulmonary arterial hypertension (PAH), Dorneles et al. [91] investigated whether ventilatory constrains contribute to intolerable dyspnoea sensations during exercise in these patients. They demonstrated that for a given rate of work during cycling, patients with PAH presented abnormal lung mechanics compared to healthy controls, manifested by higher minute ventilation, tachypnoea, decrease in inspiratory capacity and restriction of inspiratory reserve volume. They also highlighted the need for future studies aiming at investigating the effects of pharmacological and nonpharmacological interventions on lessening inspiratory constraints and, thus, dyspnoea sensations in this population.

With respect to OSA, MARILLIER et al. [92] investigated the cerebrovascular haemodynamic and oxygenation responses during exercise and in response to 8 weeks of CPAP. The study revealed that patients with OSA exhibited an insufficient increase in cerebral haemodynamic responses during exercise to the limit of tolerance that were associated with lower increases in total haemoglobin concentration. The study advanced our previous knowledge by demonstrating that restrictions in cerebral perfusion might contribute to exercise intolerance in OSA patients. The study also demonstrated that both exercise capacity 
and cerebral oxygenation remained unaffected after 8 weeks of CPAP treatment, thus indicating the need for future research in this field.

FREGONEZI et al. [93] carried out a study to ascertain whether chest wall and compartmental volume responses differ between mild asthmatic patients and healthy subjects. The study demonstrated that during mild exercise, asthma patients adopted a different breathing pattern to healthy individuals that was characterised by rapid shallow breathing, existence of end-expiratory chest wall dynamic hyperinflation, and thoraco-abdominal asynchrony between the rib cage and abdominal chest compartments. The investigators concluded that these alternations in breathing pattern may contribute to exercise limitation in mild asthmatic patients.

Regarding cystic fibrosis (CF), Di PAOLO et al. [94] highlighted the importance of assessing ventilatory efficiency $\left(V^{\prime} \mathrm{E} / V^{\prime} \mathrm{CO}_{2}\right)$ during incremental CPET. They performed a multicentre observational study comparing the slopes and intercepts of the $V^{\prime} \mathrm{E} / V^{\prime} \mathrm{CO}_{2}$ relationship, and the lowest value between $\mathrm{CF}$ patients with normal to moderate impairment in lung function and age/sex-matched healthy subjects. Ventilatory inefficiency was shown by higher values of $V^{\prime} \mathrm{E} / V^{\prime} \mathrm{CO}_{2}$ slope and nadir in CF compared to healthy individuals. This finding represented a clear feature of CF patients even among those with normal lung function. Accordingly, these indices may represent valuable outcomes for prospective trials, and for evaluating the effectiveness of different pharmacological and nonpharmacological treatments in CF. BAR-YosepH et al. [95] compared exercise capacity in mild CF, non-CF bronchiectasis and healthy controls. Although the study demonstrated that peak exercise capacity during CPET was comparable between both groups of patients and age-matched healthy controls, both mild CF and non-CF bronchiectasis patients had breathing limitations that were confirmed by low breathing reserves at the end of exercise. This should be acknowledged as a potential limitation in exercise capacity in these patients.

Finally, Dourado et al. [96] presented the findings from the cross sectional EPIMOV study, which was conducted on 1368 adults aged between 18 and 80 years old. The aim of this study was to determine, by measuring the peak oxygen uptake during CPET, the role of physical activity and cardiorespiratory fitness in the relationship between lung function with arterial hypertension [97]. This study identified only peak oxygen uptake variables as significant mediators for the relationships between lung function parameters and hypertension (Sobel-Goodman test results ranged between $60 \%$ and $79 \%$ ). The authors concluded that low cardiorespiratory fitness may provide the pathway for understanding the role of lung function as predictor of incident arterial hypertension from young adults to the elderly population.

\section{Advances in exercise pathophysiology}

This session provided new insights and interesting physiological discussions on topics that comprise the routine management of patients with COPD. All studies emphasised the importance of assessing responses to exercise in order to tackle several clinical challenges we are faced with when caring for COPD patients.

The study presented by BERTON et al. [98] sought to determine whether dual bronchodilator treatment improved exercise haemodynamics because of reduced static and dynamic hyperinflation in patients with COPD. Signal morphology impedance cardiography was used to assess cardiac output and blood flow to the quadriceps. In a double-blind crossover study in which 20 patients exercised at $75 \%$ of peak work rate after a single dose of olandaterol+tiotropium or placebo, the authors found that dual bronchodilation increased resting and peak inspiratory capacity, reduced isotime dyspnoea and improved exercise endurance, but no differences were seen either in central or peripheral haemodynamics.

The second presentation, by HaJIAN et al. [99] examined the impact of pulsed inhaled nitric oxide (iNO) on pulmonary hypertension and exercise tolerance in hypoxic COPD patients. The rationale behind this proof of concept study was that iNO would be able to cause vasodilation and reduce pulmonary artery pressures without worsening ventilation/perfusion mismatch, given that iNO would diffuse predominantly to areas of higher alveolar volume. After 4 weeks of pulsed iNO, patients showed a reduction in systolic pulmonary artery pressure and significantly increased 6MWD without worsening gas exchange. In fact, functional respiratory imaging analysis demonstrated that there was a significant association between regional ventilation and regional vasodilation during iNO therapy. While it could be argued that the lack of a familiarisation 6MWD might have overestimated the clinical response to iNO, the physiological rationale was confirmed and the results are in line with that of recent studies investigating the effects of inhaled iloprost in COPD [100].

The study presented by SouzA et al. [101] showed that NIV increases inspiratory capacity and reduces isotime dyspnoea in patients with overlapping COPD-heart failure (HF) but not in HF subjects without COPD. In addition, the use of bilevel ventilation led to longer endurance time at high-intensity exercise testing in COPD-HF subjects compared to sham ventilation, although this finding was difficult to interpret given the authors' choice of exercise protocol and the small sample size. Nevertheless, the results 
concerning improvement in symptoms and ventilatory mechanics seemed consistent and provided physiological rationale for the use of NIV in COPD-HF patients during exercise in a rehabilitation programme.

FURIAN et al. [102] presented a study showing that the acute use of dexamethasone can attenuate the increase in cerebral blood flow in response to high altitude and blunts systemic arterial pressure increase during isometric exercise compared to placebo, thus potentially preventing cerebral hyperperfusion without leading to tissue hypoxia in GOLD I-II COPD patients. In turn, BEDIN et al. [103] suggested that a peak oxygen consumption $<15 \mathrm{~mL} \cdot \mathrm{kg}^{-1} \cdot \mathrm{min}^{-1}$ predicts the occurrence of postoperative cardiopulmonary complications in patients undergoing lung resection surgery, regardless of the presence of comorbid COPD. Finally, PAPAPOSTOLOU et al. [104] retrospectively analysed demographics, pulmonary function and exercise data of $800 \mathrm{COPD}$ subjects to develop an equation that could predict peak work rate on incremental cycle ergometry. They revealed that FEV1 and diffusing capacity of the lung for carbon monoxide were the variables most strongly associated with peak work rate. When tested prospectively in a small sample of 15 GOLD II-III COPD patients, there was a good correlation between actual and predicted peak work rate but the accuracy of these estimated values was somewhat variable.

Conflict of interest: I. Almendros has nothing to disclose. S. Baillieul has nothing to disclose. C. Caballero has nothing to disclose. L.H. Degani-Costa has nothing to disclose. M. Furian has nothing to disclose. C. Hirotsu has no conflicts of interest to disclose. Z. Louvaris has nothing to disclose. S. Thiel has nothing to disclose. C.D. Turnbull reports consultancy fees outside the scope of this work from Bayer.

\section{References}

1 Moulin S, Pauly M, Thomas A, et al. Intermittent hypoxia, the hallmark of sleep apnea, induces HIF-1-dependent mitochondrial dysfunction. Eur Respir J 2018; 52: Suppl. 62, OA3285.

2 Détrait M, Bourdier G, Moulin S, et al. Mechanisms involved in the deleterious impact of intermittent hypoxia on ischemic cardiomyopathy. Eur Respir J 2018; 52: Suppl. 62, OA3286.

3 Turnbull C, Lee L, Petousi N, et al. Transcriptomic analysis identifies a unique, intermittent hypoxia mediated inflammatory profile: evidence from a cross-over randomised CPAP withdrawal trial, with and without supplemental oxygen, in OSA. Eur Respir J 2018; 52: Suppl. 62, OA3287.

4 Thiel S, Lettau F, Haile SR, et al. The effects of CPAP-withdrawal on cerebral vascular reactivity and brain oxygenation in patients with obstructive sleep apnoea: a randomised-controlled trial. Eur Respir J 2018; 52: Suppl. 62, OA3289.

5 Suarez-Giron MC, Castro-Grattoni A, Torres M, et al. Acetylsalicylic acid prevents intermittent hypoxia-induced vascular remodeling in a murine model of sleep apnea. Front Physiol 2018; 9: 600.

6 Almendros I, Menal MJ, Ozal D, et al. Differential effect of intermittent hypoxia and sleep fragmentation in the progression of Alzheimer Disease in a mouse model of obstructive sleep apnea. Eur Respir J 2018; 52: Suppl. 62, OA3284.

7 Trzepizur W, Boursier J, Le Vaillant M, et al. Independent association between obstructive sleep apnea severity and liver stiffness. Eur Respir J 2018; 52: Suppl. 62, OA3290.

8 Revol B, Jullian-Desayes I, Tamisier R, et al. Ticagrelor and central sleep apnea: analysis of VigiBase, the WHO pharmacovigilance database. Eur Respir J 2018; 52: Suppl. 62, OA3291.

9 Malhotra A, Heinzer R, Morrell MJ, et al. European prevalence of OSA in adults: Estimation using currently available data. Eur Respir J 2018; 52: Suppl. 62, OA4961.

10 Pien GW, Ye L, Keenan BT, et al. Changing faces of obstructive sleep apnea: treatment effects by cluster designation in the Icelandic Sleep Apnea Cohort. Sleep 2018; 41: https://doi.org/10.1093/sleep/zsx201.

11 Keenan BT, Kim J, Singh B, et al. Recognizable clinical subtypes of obstructive sleep apnea across international sleep centers: a cluster analysis. Sleep 2018; 41: https:/doi.org/10.1093/sleep/zsx214.

12 Ye L, Pien GW, Ratcliffe SJ, et al. The different clinical faces of obstructive sleep apnoea: a cluster analysis. Eur Respir J 2014; 44: 1600-1607.

13 Zinchuk AV, Gentry MJ, Concato J, et al. Phenotypes in obstructive sleep apnea: a definition, examples and evolution of approaches. Sleep Med Rev 2017; 35: 113-123.

14 Kim J, Keenan BT, Lim DC, et al. Symptom-based subgroups of Koreans with obstructive sleep apnea. J Clin Sleep Med 2018; 14: 437-443.

15 Anttalainen U, Hedner J, Ryan S, et al. Hypoxia and cardiovascular comorbidity in the ESADA OSA patients with insomnia symptoms. Eur Respir J 2018; 52: Suppl. 62, OA4962.

16 Johansson P, Svensson E, Alehagen U, et al. The contribution of hypoxia to the association between sleep apnoea, insomnia, and cardiovascular mortality in community-dwelling elderly with and without cardiovascular disease. Eur J Cardiovasc Nurs 2015; 14: 222-231.

17 Peker Y, Balcan B, Glantz H, et al. Polysomnographic characteristics and cardiac function in coronary artery disease patients with nonsleepy obstructive sleep apnoea. Eur Respir J 2018; 52: Suppl. 62, OA4965.

18 Balcan B, Thunström E, Peker Y. Depression and response to CPAP treatment in coronary artery disease patients with sleepy $v s$ nonsleepy obstructive sleep apnoea. Eur Respir J 2018; 52: Suppl. 62, OA4966.

19 Sun Y, Huang ZY, Sun QR, et al. CPAP therapy reduces blood pressure for patients with obstructive sleep apnoea: an update meta-analysis of randomized clinical trials. Acta Cardiol 2016; 71: 275-280.

20 Guillot M, Sforza E, Achour-Crawford E, et al. Association between severe obstructive sleep apnea and incident arterial hypertension in the older people population. Sleep Med 2013; 14: 838-842.

21 Peppard PE, Young T, Palta M, et al. Prospective study of the association between sleep-disordered breathing and hypertension. N Engl J Med 2000; 342: 1378-1384. 

longitudinal study in the general population: the Vitoria Sleep Cohort. Am J Respir Crit Care Med 2011; 184: 1299-1304.

23 O'Connor GT, Caffo B, Newman AB, et al. Prospective study of sleep-disordered breathing and hypertension: the Sleep Heart Health Study. Am J Respir Crit Care Med 2009; 179: 1159-1164.

24 Hirotsu C, Heinzer R, Marques-Vidal P, et al. Sleep determinants of incident hypertension in a population-based cohort: the CoLaus/HypnoLaus study. Eur Respir J 2018; 52: Suppl. 62, OA4963.

25 Bouloukaki I, Steiropoulos P, Bonsignore MR, et al. Mild obstructive sleep apnoea (OSA) and arterial hypertension in the EuropeanSleep Apnoea Database (ESADA) cohort study. Eur Respir J 2018; 52: Suppl. 62, OA4964.

26 Hirotsu C, Haba-Hubio J, Marques-Vidal P, et al. Obstructive sleep apnea as risk factor for incident metabolic syndrome: a multicentric prospective epidemiological study. Eur Respir J 2018; 52: Suppl. 62, OA4968.

27 Raux M, Straus C, Redolfi S, et al. Electroencephalographic evidence for pre-motor cortex activation during inspiratory loading in humans. J Physiol 2007; 578: 569-578.

28 Raux M, Ray P, Prella M, et al. Cerebral cortex activation during experimentally induced ventilator fighting in normal humans receiving noninvasive mechanical ventilation. Anesthesiology 2007; 107: 746-755.

29 Georges M, Morawiec E, Raux M, et al. Cortical drive to breathe in amyotrophic lateral sclerosis: a dyspnoea-worsening defence? Eur Respir J 2016; 47: 1818-1828.

30 Dangers L, Laviolette L, Georges M, et al. Relieving dyspnoea by non-invasive ventilation decreases pain thresholds in amyotrophic lateral sclerosis. Thorax 2017; 72: 230-235.

31 Stoeckel MC, Esser RW, Gamer M, et al. Brain responses during the anticipation of dyspnea. Neural Plast 2016; 2016: 6434987.

32 Herzog M, Sucec J, Van Diest I, et al. Observing dyspnoea in others elicits dyspnoea, negative affect and brain responses. Eur Respir J 2018; 51: 1702682.

33 Parshall MB, Schwartzstein RM, Adams L, et al. An official American Thoracic Society statement: update on the mechanisms, assessment, and management of dyspnea. Am J Respir Crit Care Med 2012; 185: 435-452.

34 Herigstad M, Faull OK, Hayen A, et al. Treating breathlessness via the brain: changes in brain activity over a course of pulmonary rehabilitation. Eur Respir J 2017; 50: 1701029.

35 von Leupoldt A. Treating anxious expectations can improve dyspnoea in patients with COPD. Eur Respir J 2017; 50: 1701352 .

36 Kent BD, Ryan S, McNicholas WT. Obstructive sleep apnea and inflammation: relationship to cardiovascular co-morbidity. Respir Physiol Neurobiol 2011; 178: 475-481.

37 Almendros I, Farre R, Torres M, et al. Early and mid-term effects of obstructive apneas in myocardial injury and inflammation. Sleep Med 2011; 12: 1037-1040.

38 Castro-Grattoni AL, Alvarez-Buve R, Torres M, et al. Intermittent hypoxia-induced cardiovascular remodeling is reversed by normoxia in a mouse model of sleep apnea. Chest 2016; 149: 1400-1408.

39 Gildeh N, Drakatos P, Higgins S, et al. Emerging co-morbidities of obstructive sleep apnea: cognition, kidney disease, and cancer. J Thorac Dis 2016; 8: E901-E917.

40 Gupta MA, Simpson FC. Obstructive sleep apnea and psychiatric disorders: a systematic review. J Clin Sleep Med 2015; 11: 165-175.

41 Emamian F, Khazaie H, Tahmasian M, et al. The association between obstructive sleep apnea and Alzheimer's disease: a meta-analysis perspective. Front Aging Neurosci 2016; 8: 78.

42 Osorio RS, Gumb T, Pirraglia E, et al. Sleep-disordered breathing advances cognitive decline in the elderly. Neurology 2015; 84: 1964-1971.

43 Lozano R, Naghavi M, Foreman K, et al. Global and regional mortality from 235 causes of death for 20 age groups in 1990 and 2010: a systematic analysis for the Global Burden of Disease Study 2010. Lancet 2012; 380: 2095-2128.

44 Vogiatzis I, Zakynthinos S. Factors limiting exercise tolerance in chronic lung diseases. Compr Physiol 2012; 2: 1779-1817.

45 Chykiamis N, Armstrong M, Spencer V, et al. Influence of the VitaBreath on exercise tolerance in patients with COPD. Eur Respir J 2018; 52: Suppl. 62, PA3361.

46 Hakamy A, Bolton CE, Gibson JE, et al. Risk of fall in patients with COPD. Thorax 2018; 73: 1079-1080.

47 Reynaud V, Muti D, Greil A, et al. Validation of the Timed Up and Go test to detect fallers in COPD patients. Eur Respir J 2018; 52: Suppl. 62, PA3366.

48 Shumway-Cook A, Brauer S, Woollacott M. Predicting the probability for falls in community-dwelling older adults using the Timed Up \& Go Test. Phys Ther 2000; 80: 896-903.

49 Barry E, Galvin R, Keogh C, et al. Is the Timed Up and Go test a useful predictor of risk of falls in community dwelling older adults: a systematic review and meta-analysis. BMC Geriatr 2014; 14: 14.

50 Weiler JM, Bonini S, Coifman R, et al. American Academy of Allergy, Asthma \& Immunology Work Group report: exercise-induced asthma. J Allergy Clin Immunol 2007; 119: 1349-1358.

51 Parsons JP, Hallstrand TS, Mastronarde JG, et al. An official American Thoracic Society clinical practice guideline: exercise-induced bronchoconstriction. Am J Respir Crit Care Med 2013; 187: 1016-1027.

52 Weiler JM, Brannan JD, Randolph CC, et al. Exercise-induced bronchoconstriction update-2016. J Allergy Clin Immunol 2016; 138: 1292-1295.

53 Bonini M, Messina J, Petrarulo S, et al. T2-endotype predicts bronchodilator (BD) response in exercise-induced bronchoconstriction (EIB). Eur Respir J 2018; 52: Suppl. 62, PA3356.

54 Oostveen E, MacLeod D, Lorino H, et al. The forced oscillation technique in clinical practice: methodology, recommendations and future developments. Eur Respir J 2003; 22: 1026-1041.

55 Seccombe L, Buddle L, Peters M, et al. Exercise induced bronchoconstriction assessed using the forced oscillation technique. Eur Respir J 2018; 52: Suppl. 62, PA3355.

56 Hammer IJ, Bovim LP, Vollsæter M, et al. Is continuous laryngoscopy during cardiopulmonary exercise testing reliable for measuring maximum oxygen uptake? Eur Respir J 2018; 52: Suppl. 62, PA3357.

57 Walsted ES, Faisal A, Pavitt MJ, et al. Laryngeal laser surgery reduces neural respiratory drive and work of breathing in exercise-induced laryngeal obstruction. Eur Respir J 2018; 52: Suppl. 62, PA3358. 

PA3362.

59 Edvardsen E, Anderssen SA, Borchsenius F, et al. Long-term effects of high-intensity training after lung cancer surgery - a five-years follow-up of a randomized controlled trial. Eur Respir J 2018; 52: Suppl. 62, PA3372. Gea J, Agusti A, Roca J. Pathophysiology of muscle dysfunction in COPD. J Appl Physiol 2013; 114: 1222-1234.

61 Levine S, Bashir MH, Clanton TL, et al. COPD elicits remodeling of the diaphragm and vastus lateralis muscles in humans. J Appl Physiol 2013; 114: 1235-1245.

62 Ramirez-Sarmiento A, Orozco-Levi M, Barreiro E, et al. Expiratory muscle endurance in chronic obstructive pulmonary disease. Thorax 2002; 57: 132-136.

63 Gea J, Pascual S, Casadevall C, et al. Muscle dysfunction in chronic obstructive pulmonary disease: update on causes and biological findings. $J$ Thorac Dis 2015; 7: E418-E438.

64 Gosselink R, Troosters T, Decramer M. Distribution of muscle weakness in patients with stable chronic obstructive pulmonary disease. J Cardiopulm Rehabil 2000; 20: 353-360.

65 Gayan-Ramirez G, Decramer M. Mechanisms of striated muscle dysfunction during acute exacerbations of COPD. J Appl Physiol 2013; 114: 1291-1299.

66 Guerri R, Gayete A, Balcells E, et al. Mass of intercostal muscles associates with risk of multiple exacerbations in COPD. Respir Med 2010; 104: 378-388.

67 Lewis $\mathrm{P}$, Sheehan D, Soares R, et al. Chronic sustained hypoxia-induced redox remodeling causes contractile dysfunction in mouse sternohyoid muscle. Front Physiol 2015; 6: 122.

68 Puig-Vilanova E, Aguilo R, Rodriguez-Fuster A, et al. Epigenetic mechanisms in respiratory muscle dysfunction of patients with chronic obstructive pulmonary disease. PLoS One 2014; 9: e111514.

69 Barreiro E, Gea J. Epigenetics and muscle dysfunction in chronic obstructive pulmonary disease. Transl Res 2015 165: 61-73.

70 Pitta F, Troosters T, Spruit MA, et al. Characteristics of physical activities in daily life in chronic obstructive pulmonary disease. Am J Respir Crit Care Med 2005; 171: 972-977.

71 O’Donnell DE, Banzett RB, Carrieri-Kohlman V, et al. Pathophysiology of dyspnea in chronic obstructive pulmonary disease: a roundtable. Proc Am Thorac Soc 2007; 4: 145-168.

72 Jolley CJ, Moxham J. A physiological model of patient-reported breathlessness during daily activities in COPD Eur Respir Rev 2009; 18: 66-79.

73 Gandevia SC, Leeper JB, McKenzie DK, et al. Discharge frequencies of parasternal intercostal and scalene motor units during breathing in normal and COPD subjects. Am J Respir Crit Care Med 1996; 153: 622-628.

74 De TA, Leeper JB, McKenzie DK, et al. Neural drive to the diaphragm in patients with severe COPD. Am J Respir Crit Care Med 1997; 155: 1335-1340.

75 Sinderby C, Spahija J, Beck J, et al. Diaphragm activation during exercise in chronic obstructive pulmonary disease. Am J Respir Crit Care Med 2001; 163: 1637-1641.

76 Jolley CJ, Luo YM, Steier J, et al. Neural respiratory drive and breathlessness in COPD. Eur Respir J 2015; 45: 355-364.

77 Schaeffer MR, Ryerson CJ, Ramsook AH, et al. Neurophysiological mechanisms of exertional dyspnoea in fibrotic interstitial lung disease. Eur Respir J 2018; 51: 1701726.

78 Demoule A, Jung B, Prodanovic H, et al. Diaphragm dysfunction on admission to the intensive care unit Prevalence, risk factors, and prognostic impact - a prospective study. Am J Respir Crit Care Med 2013; 188: 213-219.

79 Demoule A, Molinari N, Jung B, et al. Patterns of diaphragm function in critically ill patients receiving prolonged mechanical ventilation: a prospective longitudinal study. Ann Intensive Care 2016; 6: 75.

80 Jaber S, Petrof BJ, Jung B, et al. Rapidly progressive diaphragmatic weakness and injury during mechanical ventilation in humans. Am J Respir Crit Care Med 2011; 183: 364-371.

81 Dres M, Demoule A. Diaphragm dysfunction during weaning from mechanical ventilation: an underestimated phenomenon with clinical implications. Crit Care 2018; 22: 73.

82 Dres M, Goligher EC, Dube BP, et al. Diaphragm function and weaning from mechanical ventilation: an ultrasound and phrenic nerve stimulation clinical study. Ann Intensive Care 2018; 8: 53.

83 Marchioni A, Castaniere I, Tonelli R, et al. Ultrasound-assessed diaphragmatic impairment is a predictor of outcomes in patients with acute exacerbation of chronic obstructive pulmonary disease undergoing noninvasive ventilation. Crit Care 2018; 22: 109.

84 Schultz K, Jelusic D, Wittmann M, et al. Inspiratory muscle training does not improve clinical outcomes in 3-week COPD rehabilitation: results from a randomised controlled trial. Eur Respir J 2018; 51: 1702000.

85 Beaumont M, Mialon P, Le Ber C, et al. Effects of inspiratory muscle training on dyspnoea in severe COPD patients during pulmonary rehabilitation: controlled randomised trial. Eur Respir J 2018; 51: 1701107.

86 Charususin N, Gosselink R, Decramer M, et al. Randomised controlled trial of adjunctive inspiratory muscle training for patients with COPD. Thorax 2018; 73: 942-950.

87 Elkins M, Dentice R. Inspiratory muscle training facilitates weaning from mechanical ventilation among patients in the intensive care unit: a systematic review. J Physiother 2015; 61: 125-134.

88 Hoffman M, Van HM, Clerckx B, et al. Can inspiratory muscle training improve weaning outcomes in difficult to wean patients? A protocol for a randomised controlled trial (IMweanT study). BMJ Open 2018; 8: e021091.

89 Dempsey JA, Romer L, Rodman J, et al. Consequences of exercise-induced respiratory muscle work. Respir Physiol Neurobiol 2006; 151: 242-250.

90 Johnson M, Needham R, Williams R, et al. Effects of maximal volitional hyperpnoea on exercise tolerance and locomotor muscle fatigue. Eur Respir J 2018; 52: Suppl. 62, OA478.

91 Dorneles R, Plachi F, Feldmann B, et al. Sensory consequences of critical inspiratory constraints during exercise in pulmonary arterial hypertension. Eur Respir J 2018; 52: Suppl. 62, OA479.

92 Marillier M, Gruet M, Baillieul S, et al. Impaired cerebral oxygenation and exercise tolerance in patients with severe obstructive sleep apnoea syndrome. Eur Respir J 2018; 52: Suppl. 62, OA480.

93 Fregonezi G, Sarmento A, Pinto J, et al. Thoracoabdominal asynchrony contributes to exercise limitation in mild asthmatic subjects. Eur Respir J 2018; 52: Suppl. 62, OA481. 
Di Paolo M, Teopompi E, Savi D, et al. Exercise ventilatory inefficiency in adults with Cystic Fibrosis with normal to moderate impairment in lung function. Eur Respir J 2018; 52: Suppl. 62, OA483.

Bar-Yoseph R, Ilivitzki A, Gur M, et al. Exercise capacity in patients with cystic fibrosis vs non-cystic fibrosis bronchiectasis. Eur Respir J 2018; 52: Suppl. 62, OA484.

Dourado V, Almeida F, Almeida V, et al. The relationship between lung function and blood pressure and the mediation role of physical activity and cardiorespiratory fitness in adults: cross-sectional results from the EPIMOV study. Eur Respir J 2018; 52: Suppl. 62, OA482.

97 Silvestre OM, Nadruz W, Jr., Querejeta RG, et al. Declining lung function and cardiovascular risk: the ARIC study. J Am Coll Cardiol 2018; 72: 1109-1122.

98 Berton DC, Marques RD, Palmer B, et al. Effects of acute dual bronchodilator treatment (tiotropium+olodaterol) on cardiopulmonary interactions in hyperinflated patients with COPD. Eur Respir J 2018; 52: Suppl. 62, OA5340. Hajian B, Shivalkar B, Ferreira F, et al. Pulsed inhaled nitric oxide (iNO) improves exercise tolerance and shortness of breath (SOB) in severe COPD subjects with pulmonary hypertension (PH). Eur Respir J 2018; 52: Suppl. 62, OA5341

100 Wang L, Jin YZ, Zhao QH, et al. Hemodynamic and gas exchange effects of inhaled iloprost in patients with COPD and pulmonary hypertension. Int J Chron Obstruct Pulmon Dis 2017; 12: 3353-3360.

101 Souza A, Mazzuco A, Alencar MC, et al. Ventilatory assistance decreases mechanical-inspiratory constraints and improves exercise tolerance in overlapping COPD-heart failure. Eur Respir J 2018; 52: Suppl. 62, OA5342.

102 Furian M, Hartmann SE, Lichtblau M, et al. Effect of dexamethasone on cerebrovascular hemodynamics in lowlanders with COPD travelling to 3100 m. Eur Respir J 2018; 52: Suppl. 62, OA5343.

103 Bedin DCC, Ramos RP, Vieira P, et al. Is it possible to perform cardiopulmonary exercise test in laryngectomized patients? Eur Respir J 2018; 52: Suppl. 62, PA2485.

104 Papapostolou G, Jarenbäck L, Ankerst J, et al. Prediction algorithm of Wmax in COPD and evaluation by cardiopulmonary exercise testing. Eur Respir J 2018; 52: Suppl. 62, OA5345. 